Project 1010283

\title{
Biodegradation of PuEDTA and Impacts on Pu Mobility
}

\author{
Xun, Luying \\ Washington State University \\ Bolton, Jr., Harvey \\ Pacific Northwest National Laboratatory
}

\begin{abstract}
RESULTS TO DATE: This project is part of a major project (PI, Dr. Harvey Bolton, Jr. at PNNL) regarding plutonium mobility in the subsurface. Ethylenediaminetetraacetate (EDTA) is a common chelating agent that can increase the mobility of radionuclides and heavy metals in groundwater. Biodegradation of EDTA decreases the enhanced mobility. The overall objective is to understand how microbial degradation affects Plutonium-EDTA transport in the environment, and the specific objective of this component is to understand how microorganisms degrade EDTA. Over the past two years, significant progress has been made to the understanding on how EDTA is degraded by an EDTA-degrading bacterium BNC1. The characterization of EDTA and nitrilotriacetate (NTA) transport into BNC1 cells is summarized here. The uptake is the limiting step in EDTA and NTA degradation. The objectives of near-term research are described. We are making progress as projected in the proposal.
\end{abstract}

\section{A. Characterization of EDTA uptake in the EDTA-degrading bacterium BNC1}

We have previously characterized an EDTA degrading gene cluster from an EDTA-degrading bacterium BNC1. Besides catabolic genes required for EDTA degradation, four genes encoding an ABC-type transporter system was also identified. Several lines of evidence support the assignment of the ABC-type transporter as the EDTA uptake system in BNC1. Firstly, the ABC-type transporter genes and EDTA catabolic genes were transcribed as a single mRNA. Secondly, the transcription of the operon was inducible by EDTA. Thirdly, only EDTA-induced cells transported [14C]-EDTA into the cells. Fourthly, one of the transporter gene product is a periplasmic binding protein, which binds the substrate first and then transports it into the cells with the membrane proteins of the transport system. The binding protein had high affinity to Ca-EDTA and Mg-EDTA, but not for Ni-EDTA and Cu-EDTA. The binding was confirmed by both micro-calorimetric and fluorescent analyses. The results indicate that certain metal-EDTA complexes are not transported into the cell, explaining why these complexes are not used as growth substrates by BNC1. The whole cell uptake of different metal-EDTA complexes are done at PNNL by Dr. Harvey Bolton, Jr.

B. The same transport system and catabolic enzymes are responsible for both NTA and EDTA degradation in bacterium BNC1.

Several NTA-degrading bacteria have been characterized, but none of them can degrade EDTA. However, the EDTA-degrading bacterium BNC1 grows on both NTA and EDTA. We have demonstrated that the EDTA-degrading enzymes in BNC1 can also convert NTA to glycine and glyoxylate, which are common metabolic intermediates in cellular metabolism. We further determined that the periplasmic binding protein also bound Mg-NTA and Ca-NTA, but not Cu-NTA. Future research are planned to compare the gene induction by EDTA and NTA and to correlate the protein binding and whole cell transport of different metal-NTA complexes.

DELIVERABLES: Publications: 1. Bohuslavek, J., J. Payne, Y. Liu, H. Bolton, Jr., and L. Xun. 2001. Cloning, sequencing and characterization of a gene cluster involved in EDTA degradation from the bacterium BNC1. Appl. Environ. Microbiol. 67:688-695. 2. Liu, Y., T. M. Louie, J. Payne, J. Bohuslavek, H. Bolton, Jr., and L. Xun. 2001. Identification, purification and characterization of iminodiacetate oxidase from the EDTA-degrading bacterium BNC1. Appl. Environ. Microbiol. 67:696-701. 3. Characterization of EDTA uptake by the EDTA-degrading bacterium BNC1. Manuscript is preparation. 